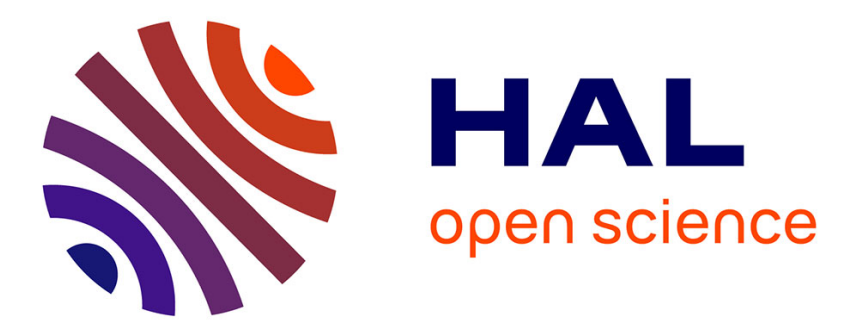

\title{
The Liquidity Regimes and the Prepayment Option of a Corporate Loan in the Finite Horizon Case
}

Timothee Papin, Gabriel Turinici

\section{To cite this version:}

Timothee Papin, Gabriel Turinici. The Liquidity Regimes and the Prepayment Option of a Corporate Loan in the Finite Horizon Case. Global Credit Review, 2015, 5 (1), pp.19-33. 10.1142/S2010493615500026 . hal-01073598v2

\section{HAL Id: hal-01073598 \\ https://hal.science/hal-01073598v2}

Submitted on 25 Jul 2015

HAL is a multi-disciplinary open access archive for the deposit and dissemination of scientific research documents, whether they are published or not. The documents may come from teaching and research institutions in France or abroad, or from public or private research centers.
L'archive ouverte pluridisciplinaire HAL, est destinée au dépôt et à la diffusion de documents scientifiques de niveau recherche, publiés ou non, émanant des établissements d'enseignement et de recherche français ou étrangers, des laboratoires publics ou privés. 


\title{
THE LIQUIDITY REGIMES AND THE PREPAYMENT OPTION OF A CORPORATE LOAN IN THE FINITE HORIZON CASE
}

\author{
TIMOTHEE PAPIN* AND GABRIEL TURINICI ${ }^{\dagger}$
}

\begin{abstract}
We investigate the prepayment option related to a corporate loan. The default intensity of the firm is supposed to follow a Cox-Ingersoll-Ross (CIR) process and the short interest rate is assumed constant. A liquidity term that represents the funding costs of the bank is introduced and modeled as a continuous time discrete state Markov jump process. The prepayment option is an American option with the payoff being an implicit function of the parameters of the problem. We give a verification result that allows to compute the price of the option. Numerical results are completely consistent with the theory; it is seen that the exercise domain may entirely disappear during such a liquidity crisis meaning that it is not optimal for the borrower to prepay. The method allows to quantify and interpret these findings.
\end{abstract}

Key words. liquidity regime, loan prepayment, mortgage option, American option, option pricing, prepayment option, CIR process, switching regimes, Markov modulated dynamics.

AMS subject classifications. 91G20, 91G30, 91G40, 91G50, 91G60, 91G80, 93E20

1. Introduction. A loan contract issued by a bank for its corporate clients often includes a prepayment option which entitles the client to pay all or a fraction of its loan earlier than the maturity. The principle of such an option is very close to the embedded option of a callable bond, although the technical part is different.

We focus here on loans with continuous dividend payment and final down-payment of the principal; the loans are affected by borrower's default in which case interests stop at the time of default and only a fraction of the principal is recovered.

When the interest rates are constant and the borrower cannot default, a simple procedure allows the borrower to decide whether the exercise of the option is worthwhile: he compares the actualized value of the remaining payments with the nominal (outstanding) value of the loan, denoted by $K$. If the former is largen than the latter then it may be optimal to prepay.

When the interest rates are not constant or borrower is subject to default, the computation of the actualization is less straightforward. It involves all possible scenarios of the interest rate and default intensity in a risk-neutral framework in order to compute the average value of remaining payments (including the final payment of the principal if applicable); this quantity will be called "PVRP" and denoted $\xi$ and is the present value of the remaining payments, i.e. the cash amount equivalent of the value of remaining payments.

To continue the evaluation of the prepayment option, the PVRP is compared with the nominal value $K$ : if $\xi \geq K$ then the borrower could prepay; note that it is not always optimal to immediately prepay when $\xi=K$ because in some situations waiting longer can be even more worthwhile for the borrower.

The bank, that proposes the loan, finances it through a bond program (possibly mutualized for several loans) at some spread depending on its own credit profile and market conditions. In order for the corporate loan to be profitable the rate of the bond, that is also indexed on LIBOR or EURIBOR, has to be lower than the rate of the loan. This condition is easy to check at the origination of both contracts and is

*BNP Paribas CIB Resource Portfolio Management and CEREMADE, Université Paris Dauphine

${ }^{\dagger}$ CEREMADE, Université Paris Dauphine (Gabriel.Turinici@dauphine.fr), PARIS 75016, FRANCE, +33144054858 
always enforced by the bank. However if the client prepays the bank finds itself in a risky situation: the periodic interest payments from client are terminated but the bank still has to pay the interests (indexed on a floating rate) and principal of its own bond; the bond does not have a prepayment option or such an option is costly. The risk is that the amount $K$ received from the client at prepayment time cannot be invested in another product with interest rate superior to that of the bond. Therefore the prepayment option is essentially a reinvestment risk and the longer the maturity of the loan, the riskier the option.

According to the normal market practice, the borrower rarely pays a premium or a penalty to prepay his/her loan. However, the prepayment risk is not negligible and the bank needs to assess it to be protected against the liquidity risk.

Thus a first question is what provisions need to be made (at the bank level) in order to handle the prepayment risk. This is an option pricing problem that can be modeled as an embedded compound American option on a risky debt owned by the borrower. As Monte-Carlo simulations are slow to converge to assess accurately the continuation value of the option during the life of the loan and that the binomial tree techniques are time-consuming for long-term loans (cf. works by D. Cossin et al. (Cossin, 2002)), we decided to focus, in this paper, on PDE version instead of binomial trees and Monte Carlo techniques.

Another crucial question is whether it is possible that many clients decide to prepay simultaneously. This may occur in a post-crisis era when clients can borrow again at lower rates. We address this question by introducing liquidity regimes to model funding costs.

Probably the most characteristic of the liquidity is that it oscillates between distinct regimes following the state of the economic environment. Between two crisis, markets are confident and it is easier for the banks to issue bonds and the liquidity market is stable. However, during a crisis, liquidity is rare and the transition between these two distinct behaviors is rarely smooth and often sudden.

In order to model the presence of distinct liquidity regimes we simulate the liquidity cost by a continuous time observable Markov chain that can have a finite list of possible values, one for each liquidity regime (see equations (3.5) and (3.6) below). It was seen in Papin and Turinici $(2013,2014)$ that considering several liquidity regimes explains better clients' prepayment behavior than a constant liquidity model.

Specific technical circumstances make the evaluation of the prepayment option less straightforward: although the goal is to value an American option the payoff of the option is highly non-standard (is dependent on the PVRP) and is close to a compound option. The characterization of the exercise region is not standard and technical conditions have to be met. Furthermore our focus here is on a specific type of dynamics (of CIR type) with even more specific interest on the situation when several regimes are present.

The most important variable throughout the paper is the borrower credit risk defined by his/her default intensity (called in the following simply "intensity"); it follows a CIR stochastic process and the liquidity cost of the bank, defined as the cost of the lender to access the cash on the market, has several distinct regimes that we model by a Markov chain. We prove a verification theorem that allows to find the price of the option and a theoretical result that allows to use rigorously an algorithm to identify the boundary of the exercise region; numerical examples are also presented.

The plan of the paper is as follows: we discuss in Section 2 related works; then we introduce the model in Section 3, followed in Sections 4 and 5 by technical properties 
of the PVRP. In Section 6 it is explained the term structure of the liquidity specific to this model. The theoretical result concerning the price of the prepayment option is given in Section 7 and the numerical results in Section 8 .

2. Literature review. Historically works that involved a prepayment option appeared in the study of mortgages, see for instance (Hillard and Slawson, 1998) and (Chen et al., 2009) for recent contributions to that literature. In that view, the prepayment option is a function depending on the interest rate and house price. Their approach is based on a binomial tree with interest rate and a house value following a CIR process.

There also exist mortgage prepayment models that use the statistical data to infer information about the prepayment, see (Schwartz and Torous, 1993). However the volume and history of data in the corporate loan market are not compatible with these methods.

Another contribution by D. Cossin et al. (Cossin, 2002) uses a binomial tree framework and is applied to corporate loans. But the approach seems to be best adapted for short maturity loans due to the inherent computational difficulties in the numerical implementation of the binomial trees.

These papers considered the practical algorithm to find a solution and did not have to consider the geometry of the exercise region because it is explicitly given by the numerical algorithm. On the contrary we have to take this into account to check the optimality of the solution and furthermore, to the best of our knowledge, none of these approaches were meant to treat several liquidity regimes. In particular the technical hypothesis (7.10) in Lemma 7.1 was never invoked in previous works on finite maturity prepayment options.

The analysis of Markov regimes has been investigated in the literature when the underlying(s) follow the Black\& Scholes dynamics with drift and volatility having Markov jumps: Guo and Zhang (Zhang and Guo, 2004) derived the closed-form solutions for vanilla American put; Guo treats in (Guo, 2001) Russian options and derives explicit solutions for the optimal stopping time; Mamon and Rodrigo find in (Mamon and Rodrigo, 2005) explicit solutions to vanilla European options. Buffington and Elliott address in (Buffington and Elliott, 2009) European and American options. Other contributions include Jobert and Rogers (2006), Yao et al. (2006), Siu et al. (2008).

A different class of contributions discuss the liquidity; among them several contributions point out that the liquidity displays "regimes" that is a finite list of distinctive macro-economic circumstances, see for instance (Dionne et al., 2011, Liang and Wang, 2012) and references within.

Works involving Markov switching regimes and CIR dynamics appears in Elliott and Siu (2009) where the bond valuation problem is considered but in their model only the mean reverting level is subject to Markov jumps. Moreover the techniques there do not apply to an American option which is our situation here. Or, as detailed below, the American option evaluation is very different from an European option and requires to check a particular hypothesis in order for the valuation to be carried over. In addition we propose a numerical method to solve the variational PDE associated to the American option.

Another related wors is Zhou and Mamon (2011) where the term structure of the interest rates is analysed. A relevant connected work is Siu (2010) where the bond price is obtained when the short rate process is governed by a Markovian regimeswitching jump-diffusion version of the Vasicek model; the authors provide in addition the suitable mathematical arguments to study piecewise Vasicek dynamics (here the 
dynamics is still piecewise but CIR).

On the other hand numerical methods are proposed in Huang et al. (2011) for the prepayment option where it is found that a fixed point policy iteration coupled with a direct control formulation seems to perform best.

Finally, we refer to Jaillet et al. (1990) for theoretical results concerning the pricing of American options in general.

The pricing of a simplified one-dimensional model was proposed in an infinite horizon (perpetual) setting in Papin and Turinici (2013). In Papin and Turinici (2014) it is investigated in addition a non-trivial dynamics of the interest rate and a numerical algorithm is proposed together with the introduction of adapted functional spaces. This paper on the contrary addresses the finite horizon case, more close to the actual practice. We also discuss the term structure of liquidity costs which is not relevant in the perpetual setting.

3. Default intensity and theoretical regime switching framework. The prepayment option is an option on the credit risk, intensity and the liquidity cost. The intensity follows a Cox-Ingersoll-Ross process (see Cox et al. (1985), Alfonsi (2005), Lamberton and Lapeyre (2008) for theoretical and numerical aspects of CIR processes and the situations where the CIR process has been used in finance and Duffie et al. (2007), Duan et al. (2012) for some alternatives):

$$
d \lambda_{t}=\gamma\left(\theta-\lambda_{t}\right) d t+\sigma \sqrt{\lambda_{t}} d W_{t}, \lambda_{0}=\overline{\lambda_{0}} .
$$

It is known (see references) that if $2 \gamma \theta \geq \sigma^{2}$ the intensity is always strictly positive.

Denote by $\mathcal{A}$ the characteristic operator (cf. (Øksendal, 2007, Chapter 7.5)) of the CIR process i.e. the operator that acts on any $C^{2}$ class function $v$ by

$$
(\mathcal{A} v)(t, \lambda)=\partial_{t} v(t, \lambda)+\gamma(\theta-\lambda) \partial_{\lambda} v(t, \lambda)+\frac{1}{2} \sigma^{2} \lambda \partial_{\lambda \lambda} v(t, \lambda) .
$$

The liquidity cost is defined as the specific cost of a bank to access the cash in a money market. This cost is modeled by a continuous time discrete space Markov chain depending on the states of the economy. We assume the interbank offered rate IBOR $r$ to be constant. We assume the economic state of the market is described by a continuous time finite state Markov chain $\mathbf{X}=\left\{X_{t}, t \geq 0\right\}$. The state space of $\mathbf{X}$ is the set of unit vectors $E=\left\{e_{1}, e_{2}, \ldots, e_{N}\right\}, e_{i}=(0, \ldots, 0,1,0, \ldots, 0)^{T} \in \mathbb{R}^{N}$. Here $T$ is the transposition operator.

Assuming the process $X_{t}$ is homogeneous in time and has a rate matrix $A$, then if $p_{t}=\mathbb{E}\left[X_{t}\right] \in \mathbb{R}^{N}$ :

$$
\begin{gathered}
\frac{d p_{t}}{d t}=A p_{t}, \\
X_{t}=X_{0}+\int_{0}^{t} A X_{u} d u+M_{t},
\end{gathered}
$$

where $\mathbf{M}=\left\{M_{t}, t \geq 0\right\}$ is a martingale with respect to the filtration generated by $\mathbf{X}$. Denote by $a_{k, j}$ the entry on the line $k$ and the column $j$ of the $N \times N$ matrix $A$ with $a_{k, j} \geq 0$ for $j \neq k$ and $\sum_{j=1}^{N} a_{k, j}=0$ for any $k$.

In differential form

$$
d X_{t}=A X_{t} d t+d M_{t}, X_{0}=\overline{X_{0}} .
$$


We denote by $l_{t} \in \mathbb{R}$ the instantaneous liquidity cost of the bank when the economy is in state $X_{t}$. To ease notations we introduce the vector $\mathcal{L}$ (containing the liquidity values for each regime) such that the liquidity values $l_{t}$ are formally a stochastic process obtained by the scalar product:

$$
l_{t}=\left\langle\mathcal{L}, X_{t}\right\rangle
$$

We consider that there is no correlation between the dynamics of the credit risk, i.e., the intensity $\lambda_{t}$, of the borrower and the cost to access the cash in a money market, i.e. the liquidity cost $l_{t}$, of the lender. Of course, this is a simplification and more general models can be considered. However this is reasonable to the first order because the two effects originate from different reasons: the liquidity is a macroeconomic variable depending on the general state of the economy; on the contrary, the default risk depends on the specific characteristics of the borrower and its business plan. See Remarks 2 and 6 for additional comments.

4. Analytical formulas for the PVRP. At the origination, the payments correspond to an interest rate, the sum of the short term interest rate (e.g., LIBOR or EURIBOR) and the contractual margin $\overline{\rho_{0}}$ chosen such that $\xi=K$. The loan has a fixed coupon corresponding to the sum of the interest rate $r$ and the initial contractual margin $\overline{\rho_{0}}$. Let $\xi(t, \lambda, X)$ be the present value of the remaining payments at time $\mathrm{t}$ of a corporate loan where $\lambda$ is the intensity at time $t, T$ is the contractual maturity, $K$ is the nominal amount, $\delta$ is the recovery rate (in the eventuality of a default) and $X_{t}$ is the state of the economy at time $t$. As the values $K, \delta$ and $T$ are fixed, we omit them from all subsequent notations.

The loan value $L V(t, \lambda, X)$ is equal to the present value of the remaining payments $\xi(t, \lambda, X)$, minus the prepayment option value denoted by $P(t, \lambda, X)$ :

$$
L V(t, \lambda, X)=\xi(t, \lambda, X)-P(t, \lambda, X)
$$

The PVRP $\xi$ is the present value of the cash flows discounted at the risky rate, where the risky rate at time $t$ is the constant risk-free rate $r$ plus the liquidity cost $l_{t}$ and the intensity $\lambda_{t}$. To describe the cash flows in a term loan we add for all $t$ all coupons $K\left(r+\overline{\rho_{0}}\right) \Delta t+O(\Delta t)$ perceived between $t$ and $t+\Delta t$ and the nominal $K$ reimbursed at the end if no default occurred (and otherwise the portion of nominal recovered $\delta \cdot K)$. The term $O(\Delta t)$ arises from the discretization error. We obtain (see also Lando (2004)):

$$
\begin{gathered}
\xi(t, \lambda, X):=\mathbb{E}\left[\int_{t}^{T}\left(K\left(r+\overline{\rho_{0}}\right)+\delta \cdot K \lambda_{\tilde{t}}\right) e^{-\int_{t}^{\tilde{t}} r+l_{u}+\lambda_{u} d u} d \tilde{t}+K e^{-\int_{t}^{T} r+l_{u}+\lambda_{u} d u}\right. \\
\left.\mid \lambda_{t}=\lambda, X_{t}=X\right]
\end{gathered}
$$

REMARK 1. The exponential weighting term in the formula above expresses, as it is classical, the fact that, for the scenarios when default occurs, the payment of the interest stops at the time of default and is replaced by a one-time payment of the recovery amount $\delta K$. 
Since the dynamics of $\lambda_{t}$ and $l_{t}$ are independent we have,

$$
\begin{aligned}
\xi(t, \lambda, X)= & K\left(r+\overline{\rho_{0}}\right) \int_{t}^{T} e^{-r(\tilde{t}-t)} \mathbb{E}\left[e^{-\int_{t}^{\tilde{t}} \lambda_{u} d u} \mid \lambda_{t}=\lambda\right] \mathbb{E}\left[e^{-\int_{t}^{\tilde{t}} l_{u} d u} \mid X_{t}=X\right] d \tilde{t} \\
& +\delta \cdot K \int_{t}^{T} e^{-r(\tilde{t}-t)} \mathbb{E}\left[\lambda_{\tilde{t}} e^{-\int_{t}^{\tilde{t}} \lambda_{u} d u} \mid \lambda_{t}=\lambda\right] \mathbb{E}\left[e^{-\int_{t}^{\tilde{t}} l_{u} d u} \mid X_{t}=X\right] d \tilde{t} \\
& +K e^{-r(T-t)} \mathbb{E}\left[e^{-\int_{t}^{T} \lambda_{u} d u} \mid \lambda_{t}=\lambda\right] \mathbb{E}\left[e^{-\int_{t}^{T} l_{u} d u} \mid X_{t}=X\right]
\end{aligned}
$$

REMARK 2. The coefficients $\gamma, \theta, \sigma$ of the CIR process do not depend on the regime $X_{t}$. Thus it is possible to separate the CIR dynamics and the Markov dynamics. A different approach can extend this result by using the properties of the PVRP as explained in Remark 6.

We obtain furthermore:

$$
\begin{aligned}
\xi(t, \lambda, X)= & K\left(r+\overline{\rho_{0}}\right) \int_{t}^{T} e^{-r(\tilde{t}-t)} B(t, \tilde{t}, \lambda) \mathbb{E}\left[e^{-\int_{t}^{\tilde{t}} l_{u} d u} \mid X_{t}=X\right] d \tilde{t} \\
& -\delta \cdot K \int_{t}^{T} e^{-r(\tilde{t}-t)} \partial_{\tilde{t}} B(t, \tilde{t}, \lambda) \mathbb{E}\left[e^{-\int_{t}^{\tilde{t}} l_{u} d u} \mid X_{t}=X\right] d \tilde{t} \\
& +K e^{-r(T-t)} B(t, T, \lambda) \mathbb{E}\left[e^{-\int_{t}^{T} l_{u} d u} \mid X_{t}=X\right]
\end{aligned}
$$

where for general $t, \tilde{t}$ we use the notation:

$$
B(t, \tilde{t}, \lambda)=\mathbb{E}\left[e^{-\int_{t}^{\tilde{t}} \lambda_{u} d u} \mid \lambda_{t}=\lambda\right] .
$$

The quantity $B(t, \tilde{t}, \lambda)$ shares the same mathematical expression with the price of a zero-coupon in a model where the short interest rates follow a CIR process. This remark allows to write (see (Cox et al., 1985, Lamberton and Lapeyre, 2008)):

$$
B(t, \tilde{t}, \lambda)=\alpha(t, \tilde{t}) e^{-\beta(t, \tilde{t}) \lambda},
$$

where,

$$
\begin{aligned}
& \alpha(t, \tilde{t})=\left(\frac{2 h e^{(\gamma+h) \frac{\tilde{t}-t}{2}}}{2 h+(\gamma+h)\left(e^{(\tilde{t}-t) h}-1\right)}\right)^{\frac{2 \gamma \theta}{\sigma^{2}}} \\
& \beta(t, \tilde{t})=\frac{2\left(e^{(\tilde{t}-t) h}-1\right)}{2 h+(\gamma+h)\left(e^{(\tilde{t}-t) h}-1\right)}, \text { where } h=\sqrt{\gamma^{2}+2 \sigma^{2}} .
\end{aligned}
$$

Obviously $B(t, \tilde{t}, \lambda)$ is monotonic with respect to $\lambda$.

In order to compute $\mathbb{E}\left[e^{-\int_{t}^{\tilde{t}} l_{u} d u} \mid X_{t}=X\right]$, we invoke the time homogeneity of the Markov chain and obtain:

$$
\mathbb{E}\left[e^{-\int_{\tilde{t}}^{t} l_{u} d u} \mid X_{\tilde{t}}=<\mathbf{X}, e_{k}>\right]=\mathbb{E}\left[e^{-\int_{0}^{t-\tilde{t}} l_{u} d u} \mid X_{\tilde{t}}=<\mathbf{X}, e_{k}>\right] .
$$

Let $f_{k}(t)$ be defined by:

$$
f_{k}(t)=\mathbb{E}\left[e^{-\int_{0}^{t} l_{u} d u} \mid X_{0}=<\mathbf{X}, e_{k}>\right] .
$$


Therefore one can write:

$$
\begin{aligned}
\xi\left(t, \lambda, e_{k}\right)= & K\left(r+\overline{\rho_{0}}\right) \int_{t}^{T} e^{-r(\tilde{t}-t)} B(t, \tilde{t}, \lambda) f_{k}(\tilde{t}-t) d \tilde{t} \\
& -\delta \cdot K \int_{t}^{T} e^{-r(\tilde{t}-t)} \partial_{t} B(t, \tilde{t}, \lambda) f_{k}(\tilde{t}-t) d \tilde{t} \\
& +K e^{-r(T-t)} B(t, T, \lambda) f_{k}(T-t) .
\end{aligned}
$$

Let $\tau$ be the time of the first jump from $X_{0}=<\mathbf{X}, e_{k}>$ to some other state. Recalling the previous definition of the parameters $a_{k, j}$ (elements of the matrix $A$ ) we know (see Lando (Lando, 2004) paragraph $7.7 \mathrm{p} 211$ ) that $\tau$ is a random variable following an exponential distribution of parameter $\alpha_{k}$ with,

$$
\alpha_{k}=\sum_{j \neq k} a_{k, j} .
$$

We also know that conditional to the fact that a jump has occurred at time $\tau$ the probability that the jump is from state $e_{k}$ to state $e_{j}$ is $p_{k, j}$, where

$$
p_{k, j}=\frac{a_{k, j}}{\alpha_{k}} .
$$

Thus,

$$
\begin{gathered}
f_{k}(t)=\mathbb{P}(\tau>t) e^{-\mathcal{L}_{k} t}+\mathbb{E}\left[\mathbb{P}(\tau \leq t) e^{-\mathcal{L}_{k} \tau} \sum_{j \neq k} \mathbb{P}\left(l_{\tau}=\mathcal{L}_{j}\right) \mathbb{E}\left[e^{-\int_{\tau}^{t} l_{u} d u} \mid X_{\tau}=<\mathbf{X}, e_{j}>\right]\right] \\
=e^{-\left(\mathcal{L}_{k}+\alpha_{k}\right) t}+\alpha_{k} \int_{0}^{t} e^{-\left(\mathcal{L}_{k}+\alpha_{k}\right) \tau} \sum_{j \neq k} p_{k, j} f_{j}(t-\tau) d \tau .
\end{gathered}
$$

Then,

$$
\begin{gathered}
e^{\left(\mathcal{L}_{k}+\alpha_{k}\right) t} f_{k}(t)=1+\alpha_{k} \int_{0}^{t} e^{\left(\mathcal{L}_{k}+\alpha_{k}\right)(t-\tau)} \sum_{j \neq k} p_{k, j} f_{j}(t-\tau) d \tau \\
=1+\alpha_{k} \int_{0}^{t} e^{\left(\mathcal{L}_{k}+\alpha_{k}\right) s} \sum_{j \neq k} p_{k, j} f_{j}(s) d s
\end{gathered}
$$

By differentiation with respect to $t$ :

$$
\frac{d}{d t}\left[e^{\left(\mathcal{L}_{k}+\alpha_{k}\right) t} f_{k}(t)\right]=\alpha_{k} e^{\left(\mathcal{L}_{k}+\alpha_{k}\right) t} \sum_{j \neq k} p_{k, j} f_{j}(t) .
$$

Then

$$
\frac{d f_{k}(t)}{d t}+\left(\mathcal{L}_{k}+\alpha_{k}\right) f_{k}(t)=\alpha_{k} \sum_{j \neq k} p_{k, j} f_{j}(t)
$$

Thus,

$$
\frac{d f_{k}(t)}{d t}=\left[\sum_{j \neq k} \alpha_{k} p_{k, j} f_{j}(t)\right]-\left(\mathcal{L}_{k}+\alpha_{k}\right) f_{k}(t) .
$$

Denote $F(t)=\left(f_{1}(t), f_{2}(t), \ldots, f_{N}(t)\right)^{T}$ and introduce the $N \times N$ matrix $B$,

$$
B_{i, j}=\left\{\begin{array}{ccc}
\alpha_{i} p_{i, j} & \text { if } & i \neq j \\
-\left(\alpha_{i}+\mathcal{L}_{i}\right) & \text { if } & i=j
\end{array}\right.
$$


From equation (4.13) we obtain,

$$
\begin{gathered}
\frac{d F(t)}{d t}=B F(t) \text { thus } F(t)=e^{B t} F(0), \\
F(0)=\left(f_{k}(0)\right)_{k=1}^{N}=(1,1, \ldots, 1)^{T} \in \mathbb{R}^{N} .
\end{gathered}
$$

We have therefore analytical formulas for the PVRP $\xi(t, \lambda, X)$. We refer the reader to Elliott and Siu (2009) for similar considerations on a related CIR switching dynamics.

REMARK 3. When all liquidity parameters $\mathcal{L}_{k}$ are equal (to some quantity $l$ ) then $B=A-l \cdot I d$ (here Id is the identity matrix) and then we obtain (after some computations) that $f_{k}(t)=e^{-l t}$ thus the payoff is equal to that of a one-regime dynamics with interest rate $r+l$, which is consistent with intuitive image we may have. Another limiting case is when the switching is very fast.

The margin $\overline{\rho_{0}}$ is set to satisfy the equilibrium equation:

$$
\xi\left(0, \overline{\lambda_{0}}, \overline{X_{0}}\right)=K,
$$

which expresses the fact that the present value of the future cash flows (weighted consistently with the probability of survival) is equal to the nominal $K$. Therefore according to (4.10) if $\overline{X_{0}}=<\mathbf{X}, e_{k_{0}}>$,

$$
\overline{\rho_{0}}=\frac{1+\delta \int_{t}^{T} e^{-r(\tilde{t}-t)} \partial_{\tilde{t}} B(t, \tilde{t}, \lambda) f_{k_{0}}(\tilde{t}-t) d \tilde{t}-e^{-r(T-t)} B(t, T, \lambda) f_{k_{0}}(T-t)}{\int_{t}^{T} e^{-r(\tilde{t}-t)} B(t, \tilde{t}, \lambda) f_{k_{0}}(\tilde{t}-t) d \tilde{t}}-r .
$$

REMARK 4. Note that we assume no additional commercial margin. If an additional commercial margin $\mu_{0}$ is to be considered then $\overline{\rho_{0}}$ is first computed as above and then replaced by $\overline{\rho_{0}}+\mu_{0}$ in Equation (4.2). Equation (4.17) still holds with some adapted value instead of $\overline{\lambda_{0}}$. With these changes all results in the paper are valid, with the provision that the price of the prepayment option is $P\left(0, \overline{\lambda_{0}}, \overline{X_{0}}\right)$.

We will also need to introduce for any $k=1, \ldots, N$ the (parity boundary) function $\bar{\Lambda}_{k}^{0}(t)$ such that

$$
\xi\left(t, \bar{\Lambda}_{k}^{0}(t), e_{k}\right)=K, \quad \forall t \in[0, T[.
$$

Of course, $\bar{\Lambda}_{\overline{X_{0}}}^{0}(0)=\overline{\lambda_{0}}$. Moreover $\forall t \in[0, T], \xi\left(t, \lambda, e_{k}\right)$ is decreasing with respect to $\lambda$; when $\xi\left(t, 0, e_{k}\right)<K$ there is no solution to eqn. (4.17) and we will chose by convention $\bar{\Lambda}_{k}^{0}(t)=0$. This can happen for a regime different from $\overline{X_{0}}$ (the initial regime) when the PVRP value is always less than the parity, see also latter the numerical application. In this case there is never a parity boundary in this regime and no exercise. We put $\bar{\Lambda}_{k}^{0}(t)=0$ just as a mathematical convention (which allows to unify the mathematical formulas and not distinguish such a case separately).

Remark 5. The value $\bar{\Lambda}_{k}^{0}(T)=\left.\bar{\Lambda}_{k}^{0}(t)\right|_{t=T}$ is not defined because

$$
\xi\left(T, \lambda, e_{k}\right)=K, \forall \lambda \in \mathbb{R}^{+}, \forall k .
$$


5. Further properties of the PVRP $\xi$. We also introduce below a PDE formulation for $\xi$. To ease the notations we introduce the operator $\mathcal{A}^{\mathcal{R}}$ that acts on functions $v(t, \lambda, X)$ as follows:

$$
\left(\mathcal{A}^{\mathcal{R}} v\right)\left(t, \lambda, e_{k}\right)=(\mathcal{A} v)\left(t, \lambda, e_{k}\right)-\left(r+\mathcal{L}_{k}+\lambda\right) v\left(t, \lambda, e_{k}\right)+\sum_{j=1}^{N} a_{k, j}\left(v\left(t, \lambda, e_{j}\right)-v\left(t, \lambda, e_{k}\right)\right) .
$$

Having defined the dynamics (3.1) and (3.5) one can use an adapted version of the Feynman-Kac formula (in the same way as in Elliott and Siu (2009), Siu (2010), Zhang and Guo (2004)) in order to conclude that PVRP defined by (4.2) satisfies the equation:

$$
\left\{\begin{array}{l}
\left(\mathcal{A}^{\mathcal{R}} \xi\right)\left(t, \lambda, e_{k}\right)+\left(\delta \cdot \lambda+r+\overline{\rho_{0}}\right) K=0, \\
\xi\left(T, \lambda, e_{k}\right)=K, \quad \forall \lambda>0 \text { and } \forall e_{k} \in E .
\end{array}\right.
$$

REMARK 6. When the coefficients $\gamma, \theta, \sigma$ of the CIR process depend on the regime (see Remark 2) the analytical formulas in the previous section do not work any more; however the PDE formulation will work with the operator $\mathcal{A}^{\mathcal{R}}$ in Equation (5.2) replaced by the operator $\mathcal{A}_{k}^{\mathcal{R}}$ defined as:

$$
\begin{aligned}
& \mathcal{A}_{k}^{\mathcal{R}}(v)\left(t, \lambda, e_{k}\right)=\partial_{t} v\left(t, \lambda, e_{k}\right)+\gamma_{k}\left(\theta_{k}-\lambda\right) \partial_{\lambda} v\left(t, \lambda, e_{k}\right)+\frac{1}{2} \sigma_{k}^{2} \lambda \partial_{\lambda \lambda} v\left(t, \lambda, e_{k}\right) \\
& -\left(r+\mathcal{L}_{k}+\lambda\right) v\left(t, \lambda, e_{k}\right)+\sum_{j=1}^{N} a_{k, j}\left(v\left(t, \lambda, e_{j}\right)-v\left(t, \lambda, e_{k}\right)\right)
\end{aligned}
$$

See also the discussion in Remark 8 for the boundary conditions.

6. Term-structure of the liquidity cost. The continuous time Markov chain allows to define the liquidity cost of the bank to access the cash on the market according to several distinct regimes. Therefore in each regime we can build a term-structure of the liquidity cost that represents the cost at different terms (time horizons). The term-structure curve of the liquidity cost can be compared to a term structure curve for the interest rates. In the more stressful regime, the curve will be inverted (convex) and the short maturities have a higher price than medium-term maturities. It is the rarest type of curve and is observed during an economic recession (see Figure 8.1). The liquidity $\operatorname{cost} L_{t, T}$ for a contractual maturity $T$ at time $t$ is defined by the following equality:

$$
e^{-L_{t, T} \cdot(T-t)}=\mathbb{E}\left[e^{-\int_{t}^{T} l_{u} d u} \mid X_{t}=<\mathbf{X}, e_{k}>\right]
$$

Therefore,

$$
L_{t, T}=-\frac{\ln \left(f_{k}(T-t)\right)}{T-t} .
$$

7. Valuation of the prepayment option. The valuation problem of the prepayment option is equivalent to that of an American call option on a risky debt owned by the borrower. It is not a vanilla option but rather close in principle to a compound product because the payoff is at its turn a contingent claim. The prepayment option allows borrower to buy back and refinance his debt according to his updated credit profile at any time during the life of the option. 
As discussed above, the prepayment exercise results in a pay-off $(\xi(t, \lambda)-K)^{+}$ for the borrower. The option is therefore an American option with pay-off:

$$
\chi(t, \lambda, X)=(\xi(t, \lambda, X)-K)^{+} .
$$

The following result allows to compute the price of the prepayment option.

THEOREM 7. For each function $\Lambda:[0, T] \rightarrow\left(\mathbb{R}_{+}\right)^{N}$ which is $C^{2}$ on $[0, T[$ and such that the domain $\{(t, \lambda) \mid t \in] 0, T\left[, \lambda>\Lambda_{k}(t)\right\}$ is locally Lipschitz for any $k=1, \ldots, N$, define $P_{\Lambda}(t, \lambda, X)$ such that:

$$
\begin{aligned}
& P_{\Lambda}\left(\cdot, \cdot, e_{k}\right) \text { is Lipschitz continuous on }[0, T] \times[0, \infty[, \forall k \\
& P_{\Lambda}\left(t, \lambda, e_{k}\right)=\chi\left(t, \lambda, e_{k}\right), \forall \lambda \in\left[0, \Lambda_{k}(t)\right], t \in[0, T] \\
& \left.\left(\mathcal{A}^{\mathcal{R}} P_{\Lambda}\right)\left(t, \lambda, e_{k}\right)=0, \forall \lambda>\Lambda_{k}(t), t \in\right] 0, T[k=1, \ldots, N \\
& \lim _{\lambda \rightarrow \infty} P_{\Lambda}\left(t, \lambda, e_{k}\right)=0, \quad k=1, \ldots, N, t \in[0, T] \\
& P_{\Lambda}\left(T, \lambda, e_{k}\right)=0, k=1, \ldots, N \text { and } \forall \lambda>0 .
\end{aligned}
$$

Suppose a function $\Lambda^{*}:[0, T] \rightarrow\left(\mathbb{R}_{+}\right)^{N}$ (satisfying same hypotheses as above) exists such that $\Lambda^{*}(t) \in \prod_{k=1}^{N}\left[0,\left(\overline{\rho_{0}}-\mathcal{L}_{k}\right)^{+} \wedge \bar{\Lambda}_{k}^{0}(t)\right]$ and for all $k=1, \ldots, N$ and $\left.\forall t \in\right] 0, T[$ :

$$
\begin{aligned}
& \left\|\frac{d \Lambda^{*}(t)}{d t}\right\| \leq \infty, \forall t<T, \forall \lambda, \\
& P_{\Lambda^{*}}(t, \lambda, X) \geq \chi(t, \lambda, X) \forall t, \lambda, X, \\
& \left.\frac{\partial P_{\Lambda^{*}}\left(t, \lambda, e_{k}\right)}{\partial \lambda}\right|_{\lambda=\left(\Lambda_{k}^{*}(t)\right)^{+}}=\left.\frac{\partial \chi\left(t, \lambda, e_{k}\right)}{\partial \lambda}\right|_{\lambda=\left(\Lambda_{k}^{*}(t)\right)^{-}} \text {if } \Lambda_{k}^{*}(t)>0, \\
& \sum_{j=1}^{N} a_{k, j}\left(P_{\Lambda^{*}}\left(t, \lambda, e_{j}\right)-\chi\left(t, \lambda, e_{j}\right)\right)+K\left(\mathcal{L}_{k}+\lambda(1-\delta)-\overline{\rho_{0}}\right) \leq 0, \\
& \forall \lambda \in] \min _{j} \Lambda_{j}^{*}(t), \Lambda_{k}^{*}(t)[.
\end{aligned}
$$

Then $P=P_{\Lambda^{*}}$.

The Theorem can be rephrased as follows: for each candidate for the exercise boundary $\Lambda$ one can construct a price candidate $P_{\Lambda}$; the real price will correspond to the optimal boundary $\Lambda^{*}$ which is the one that fulfills the conditions listed in the second part of the Theorem. In the exercise region the price has to be equal to the payoff. However in the non-exercise region the price is only given by a PDE since in this region the exercise value is always higher than the payoff because more is gained from waiting than from exercising. The PDE reflects the local nature of the properties of the price.

REMARK 8. Any American option satisfies a PDE equation in its continuation part, which is here equation (7.4). The equation depends only on the dynamics of the underlying because it uses the generator (here $\mathcal{A}^{\mathcal{R}}$ ) of the stochastic process. The Equation (7.4) is to be interpreted in the sense of viscosity solutions, see Crandall et al. (1992) for an introduction, Barles and Burdeau (1995) for a treatment of degenerate PDEs and Achdou and Pironneau (2005) for an explanation of how the introduction of weighted Sobolev spaces can also help to give a meaning to this equation. When some $\Lambda_{k}^{*}(t)=0$ no boundary conditions are needed at $\lambda=0$ as the solution will select by itself the right value. The requirement (7.2) selects an unique solution to (7.3)-(7.6).

On the contrary, equations such as (7.9) and (7.10) depend on our proof strategy (see Lemma 7.1). 
Proof. The Theorem is a verification result. As such we suppose that a "solution candidate" is presented to us that satisfies all hypothesis and we prove that this is indeed the price of the option. This is proved in the form of two inequalities: first we prove that the candidate is a lower bound for the price, then we prove that it is a upper bound for the price. The first implication is more straightforward because the price is a supremum over all stopping times. The second implication is more involved and requires the use of Lemma 7.1.

The valuation of an American option related the admissible trading and consumptions strategies to the price $P(t, \lambda, X)$ of the prepayment option by computing an optimal stopping time associated to the pay-off. Denote by $\mathcal{T}_{t, T}$ the ensemble of stopping times between $t$ and $T$, then (see Musiela and Rutkowski (2005, Chapter 5) and also Myneni (1992), Lamberton and Lapeyre (2008)):

$$
P(t, \lambda, X)=\sup _{\tau \in \mathcal{T}_{t, T}} \mathbb{E}\left[e^{-\int_{t}^{\tau} r+l_{u}+\lambda_{u} d u} \chi\left(t, \lambda_{\tau}, X_{\tau}\right) \mid \lambda_{t}=\lambda, X_{t}=X\right] .
$$

We note that if $\tau_{\Lambda}$ is the stopping time associated with exiting the domain

$$
\bigcup_{k=1}^{N}\left\{(t, \lambda) \mid \lambda>\Lambda_{k}(t), t \leq T\right\},
$$

then:

$$
P_{\Lambda}(t, \lambda, X)=\mathbb{E}\left[e^{-\int_{0}^{\tau_{\Lambda}} r+l_{u}+\lambda_{u} d u} \chi\left(t, \lambda_{\tau_{\Lambda}}, X_{\tau_{\Lambda}}\right) \mid \lambda_{t}=\lambda, X_{t}=X\right] .
$$

Thus for any $\Lambda$ we have $P \geq P_{\Lambda}$; when $\Lambda$ has some null coordinates the continuity (ensured among others by the Remark 8 ) shows that we still have $P \geq P_{\Lambda}$. In particular for $\Lambda^{*}$ we obtain $P \geq P_{\Lambda^{*}}$; all that remains to be proved is the reverse inequality i.e. $P \leq P_{\Lambda^{*}}$.

Note first that since $\Lambda^{*}$ is a $C^{2}$ function the curve $\Lambda^{*}(t)$ is $C^{2}$ and thus in particular the tangent is defined at any point. Both $P_{\Lambda^{*}}$ and $\chi$ are $C^{2}$ on each subdomain delimited by $\Lambda^{*}(t)$ and in particular condition (7.3) implies that the derivatives of $P_{\Lambda^{*}}\left(\cdot, \cdot, e_{k}\right)$ and $\chi\left(\cdot, \cdot, e_{k}\right)$ match along the tangent to $\Lambda_{k}^{*}(t)$ (for any $k$ ). The condition (7.7) implies that the direction of the $\lambda$ axis is linearly independent from the tangent to $\Lambda_{k}^{*}(t)$ and since the derivatives of $P_{\Lambda^{*}}\left(\cdot, \cdot, e_{k}\right)$ and $\chi\left(\cdot, \cdot, e_{k}\right)$ match also along the direction of the $\lambda$ axis this means that they are equal in any direction. Therefore for any $k$ the function $P_{\Lambda^{*}}\left(\cdot, \cdot, e_{k}\right)$ is of $C^{1}$ class on the whole domain.

Now we follow the technique in Thm. 10.4.1 (Øksendal, 2007, Section 10.4 page 227) (see also Zhang and Guo (2004) for similar considerations). First one can use the same arguments as in Øksendal (2007, Appendix D) and work as if $P_{\Lambda^{*}}$ is $C^{2}$ (not only $C^{1}$ as the hypothesis ensures).

Denote $\mathcal{D}_{\Lambda^{*}}=\bigcup_{k=1}^{N}\left\{\left(t, \lambda, e_{k}\right) \mid \lambda \in\left[0, \Lambda_{k}^{*}(t)\right]\right\}$ (this will be the exercise region) and $\mathcal{C}_{\Lambda^{*}}=\mathbb{R}_{+}^{2} \times E \backslash \mathcal{D}_{\Lambda^{*}}$ (this will be the continuation region).

The Lemma 7.1 proves that $\mathcal{A}^{\mathcal{R}} P_{\Lambda^{*}}$ is non-positive everywhere (and is null on $\left.\mathcal{C}_{\Lambda^{*}}\right)$. The Itto formula allows to write:

$$
\left.\left.d\left(e^{-\int_{0}^{t} r+l_{s}+\lambda_{s} d s} P_{\Lambda^{*}}\left(t, \lambda_{t}, X_{t}\right)\right)\right)=e^{-\int_{0}^{t} r+l_{s}+\lambda_{s} d s}\left(\mathcal{A}^{\mathcal{R}} P_{\Lambda^{*}}\right)\left(t, \lambda_{t}, X_{t}\right)\right) d t+d Y_{t},
$$


where $Y_{t}$ is a martingale. Taking averages and integrating from 0 to a stopping time $\tau$ it follows from $\mathcal{A}^{\mathcal{R}} P_{\Lambda^{*}} \leq 0$ that:

$$
\begin{aligned}
& P_{\Lambda^{*}}(t, \lambda, X) \geq \mathbb{E}\left[e^{-\int_{0}^{\tau} r+l_{u}+\lambda_{u} d u} P_{\Lambda^{*}}\left(t, \lambda_{\tau}, X_{\tau}\right) \mid \lambda_{0}=\lambda, X_{0}=X\right] \\
& \geq \mathbb{E}\left[e^{-\int_{0}^{\tau} r+l_{u}+\lambda_{u} d u} \chi\left(t, \lambda_{\tau}, X_{\tau}\right) \mid \lambda_{0}=\lambda, X_{0}=X\right] .
\end{aligned}
$$

Since this is true for any stopping time $\tau$ the conclusion follows.

REMARK 9. An alternative proof is to recall that the price $P$ is the unique solution of the following quasi-variational inequality, see Bensoussan and Lions (1982), Bardi and Capuzzo-Dolcetta (1997):

$$
\max \left\{\mathcal{A}^{\mathcal{R}} P, \chi-P\right\}=0 .
$$

But Lemma 7.1 and hypothesis (7.8) imply that $P_{\Lambda^{*}}$ is also a solution of this problem. By uniqueness $P=P_{\Lambda^{*}}$. In this approach one has nevertheless to check the technical points related to the viscosity interpretation of the equation for the price: formal definition of the quasi-variational inequality, equivalence with the price, uniqueness of the solution, a priori Lipschitz regularity of the price.

LEMмa 7.1. Under the hypothesis of the Thm. 7 the following inequality holds (strongly except for the values $(t, \lambda, X)=\left(t, \Lambda_{j}^{*}, e_{k}\right)$ where it holds in the viscosity sense):

$$
\left(\mathcal{A}^{\mathcal{R}} P_{\Lambda^{*}}\right)(t, \lambda, X) \leq 0, \forall \lambda>0, \forall X
$$

Proof.

The result is needed for the proof of the previous Theorem. The non-trivial part of this lemma comes from the fact that if for fixed $k$ we have for $\lambda$ in a neighborhood of some $\lambda_{1}$ : $P_{\Lambda^{*}}\left(t, \lambda, e_{k}\right)=\chi\left(t, \lambda, e_{k}\right)$ this does not necessarily imply $\left(\mathcal{A}^{\mathcal{R}} P_{\Lambda^{*}}\right)\left(t, \lambda_{1}, e_{k}\right)=\left(\mathcal{A}^{\mathcal{R}} \chi\right)\left(t, \lambda_{1}, e_{k}\right)$ because $\mathcal{A}^{\mathcal{R}}$ depends on other values $P_{\Lambda^{*}}\left(t, \lambda, e_{j}\right)$ with $j \neq k$.

From (7.4) the conclusion is immediately verified for $X=e_{k}$ for any $\left.\lambda \in\right] \Lambda_{k}^{*}(t), \infty[$.

We now treat the alternative $\lambda<\min _{j} \Lambda_{j}^{*}(t)$; in particular it follows that $0 \leq$ $\lambda<\min _{j} \Lambda_{j}^{*}(t) \leq \bar{\Lambda}_{\ell}^{0}(t)$ for any $\ell$ thus $\bar{\Lambda}_{\ell}^{0}(t)>0$. Note that $\Lambda_{k}^{*}(t)<\bar{\Lambda}_{k}^{0}(t)$ implies $\xi\left(t, \Lambda_{k}^{*}(t), e_{k}\right) \geq \xi\left(t, \bar{\Lambda}_{k}^{0}(t), e_{k}\right)=K$ for any $k=1, \ldots, N$ thus $\chi\left(t, \lambda, e_{k}\right)=\xi\left(t, \lambda, e_{k}\right)-$ $K$ for any $\lambda \in\left[0, \Lambda_{k}^{*}(t)\right]$ and any $k$. Furthermore since $\lambda<\min _{j} \Lambda_{j}^{*}(t)$ we obtain $P_{\Lambda^{*}}\left(t, \lambda, e_{k}\right)=\chi\left(t, \lambda, e_{k}\right)=\xi\left(t, \lambda, e_{k}\right)-K$ for any $k$. Fix $X=e_{k}$; then:

$$
\begin{aligned}
& \left(\mathcal{A}^{\mathcal{R}} P_{\Lambda^{*}}\right)\left(t, \lambda, e_{k}\right)=\left(\mathcal{A}^{\mathcal{R}} \chi\right)\left(t, \lambda, e_{k}\right) \\
& =\left(\mathcal{A}^{\mathcal{R}}(\xi-K)\right)\left(t, \lambda, e_{k}\right) \\
& =\left(\mathcal{A}^{\mathcal{R}} \xi\right)\left(t, \lambda, e_{k}\right)-\mathcal{A}^{\mathcal{R}}(K) \\
& =-\left(\delta \cdot \lambda+r+\overline{\rho_{0}}\right) K+\left(r+\mathcal{L}_{k}+\lambda\right) K \\
& =K\left(\mathcal{L}_{k}+(1-\delta) \lambda-\overline{\rho_{0}}\right) \\
& \leq K\left(\mathcal{L}_{k}+(1-\delta) \Lambda_{k}^{*}(t)-\overline{\rho_{0}}\right) \leq 0
\end{aligned}
$$

the last inequality being true by hypothesis.

The last alternative is $\lambda \in] \min _{j} \Lambda_{j}^{*}(t), \Lambda_{k}^{*}(t)\left[\right.$; in this case $P_{\Lambda^{*}}\left(t, \lambda, e_{k}\right)=\chi\left(t, \lambda, e_{k}\right)$ but some terms $P_{\Lambda^{*}}\left(t, \lambda, e_{j}\right)$ for $j \neq k$ may differ from $\chi\left(t, \lambda, e_{j}\right)$. This point arises 
because the payoff $\chi$ itself has a complex structure and the technical condition to be satisfied was not treated in previous works (see for instance Zhang and Guo (2004)).

Recalling the properties of $\xi$ one obtains (and since $P_{\Lambda^{*}}\left(t, \lambda, e_{k}\right)=\chi\left(t, \lambda, e_{k}\right)$ ):

$$
\begin{aligned}
& \left(\mathcal{A}^{\mathcal{R}} P_{\Lambda^{*}}\right)\left(t, \lambda, e_{k}\right)=(\mathcal{A} \chi)\left(t, \lambda, e_{k}\right)-\left(r+\mathcal{L}_{k}+\lambda\right) \chi\left(t, \lambda, e_{k}\right)+\sum_{j=1}^{N} a_{k, j}\left(P_{\Lambda^{*}}\left(t, \lambda, e_{j}\right)-\chi\left(t, \lambda, e_{k}\right)\right) \\
& =\left(\mathcal{A}^{\mathcal{R}} \chi\right)\left(t, \lambda, e_{k}\right)+\sum_{j=1}^{N} a_{k, j}\left(P_{\Lambda^{*}}\left(t, \lambda, e_{j}\right)-\chi\left(t, \lambda, e_{j}\right)\right) \\
& =\left(\mathcal{A}^{\mathcal{R}} \xi\right)\left(t, \lambda, e_{k}\right)-\mathcal{A}^{\mathcal{R}}(K)+\sum_{j=1}^{N} a_{k, j}\left(P_{\Lambda^{*}}\left(t, \lambda, e_{j}\right)-\chi\left(t, \lambda, e_{j}\right)\right) \\
& =-K\left(\delta \cdot \lambda+r+\overline{\rho_{0}}\right)+\left(r+\mathcal{L}_{k}+\lambda\right) K+\sum_{j=1}^{N} a_{k, j}\left(P_{\Lambda^{*}}\left(t, \lambda, e_{j}\right)-\chi\left(t, \lambda, e_{j}\right)\right) \leq 0
\end{aligned}
$$

where for the last inequality we use hypothesis (7.10). Finally, since we proved that $\left(\mathcal{A}^{\mathcal{R}} P_{\Lambda^{*}}\right)(t, \lambda, X) \leq 0$ strongly except for the values $(t, \lambda, X)=\left(t, \Lambda_{j}^{*}(t), e_{k}\right)$ and since $P_{\Lambda^{*}}$ is of $C^{1}$ class we obtain the conclusion.

REMARK 10. Several remarks are relevant here:

1. when $N>1$ checking (7.10) does not involve any computation of derivatives and is straightforward.

2. the Theorem is a verification result i.e., only gives sufficient conditions for a candidate to be the option price. However results in the literature (see Chen and Chadam (2007) and references within) indicate that the boundary will probably be even more regular, $C^{\infty}$ on $[0, T[$. The behaviour near final time $T$ is not expected to be singular with respect to $\lambda$ (because there is no singularity in the payoff function there) but we do not exclude that $\lim _{t \uparrow T} \frac{d \Lambda_{k}^{*}(t)}{d t}=\infty$ which is equivalent to say that the derivative with respect to $\lambda$ of the inverse of $t \mapsto \Lambda_{k}^{*}(t)$ is null.

3. The condition (7.10) is a sufficient technical hypothesis. Its role is to ensure that the solution has local optimality properties, i.e., waiting beyond the optimal exercise time is not optimal.

8. Numerical Application. The numerical resolution of the partial differential equation (7.4) is required in order to compute the value of the prepayment option. We describe below the use of a finite difference method as discretization choice, but some cases may require different treatment.

To avoid working with an infinite domain we truncate at $\lambda_{\max }$. Then a boundary condition is imposed on $\lambda_{\max }$ which leads to a numerical problem posed in the finite domain $\cup_{k=1}^{N}\left\{(t, \lambda) \mid \lambda \in\left[\Lambda_{k}(t), \lambda_{\max }\right]\right\}$.

We introduce the time step $\Delta t$ and space step $\Delta \lambda$ and look for an approximation $P_{\ell, k}^{n}$ of $P_{\Lambda}\left(n \Delta t, \ell \Delta \lambda, e_{k}\right)$. The first and second derivative are approximated by (centered) finite difference formula and the time propagation by a Crank-Nicholson 
scheme:

$$
\begin{aligned}
& \frac{P_{\ell, k}^{n+1}-P_{\ell, k}^{n}}{\Delta t}+\frac{\gamma(\theta-(\ell \Delta \lambda))}{2}\left[\frac{P_{\ell+1, k}^{n+1}-P_{\ell-1, k}^{n+1}}{2 \Delta \lambda}+\frac{P_{\ell+1, k}^{n}-P_{\ell-1, k}^{n}}{2 \Delta \lambda}\right] \\
& +\frac{\sigma^{2}}{4}(\ell \Delta \lambda)\left[\frac{P_{\ell+1, k}^{n+1}-2 P_{\ell, k}^{n+1}+P_{\ell-1, k}^{n+1}}{\Delta \lambda^{2}}+\frac{P_{\ell+1, k}^{n}-2 P_{\ell, k}^{n}+P_{\ell-1, k}^{n}}{\Delta \lambda^{2}}\right] \\
& -\left(r+\mathcal{L}_{k}+(\ell \Delta \lambda)\right) \frac{P_{\ell, k}^{n+1}+P_{\ell, k}^{n}}{2}+\sum_{j=1}^{N} a_{k, j}\left[\frac{P_{\ell, j}^{n+1}-P_{\ell, k}^{n+1}}{2}+\frac{P_{\ell, j}^{n}-P_{\ell, k}^{n}}{2}\right]=0
\end{aligned}
$$

At each time step the advancement of this implicit time stepping scheme required the resolution of one linear system. A standard computation shows that the truncation error of this scheme is $O\left(\Delta t^{2}+\Delta \lambda^{2}\right)$, see Press et al. (2007) for details. This truncation arises from the use of time steps $\Delta t$ and $\Delta \lambda$. Our implementation of this finite difference method is written in $M A T L A B^{\odot}$. As the method is implicit it is unconditionally stable for our setting.

See also Remark 8 for the situation when some $\Lambda_{k}(t)$ is null: there the PDE for regime $e_{k}$ is defined over the full semi-axis $\lambda>0$ and it is never optimal to exercise in this regime. The PDE is defined with homogeneous boundary conditions at $\lambda_{\max }$ (or Neumann, see below) and without any boundary conditions at $\lambda=0$. To ensure the same number of equations and unknowns the equation is discretized at $\lambda=0$ too but the second order derivative is null there. Only first order terms and a first order derivative remain. The first order derivative is discretized with a lateral second order finite difference formula that involves only the function values at $\lambda=0, \Delta \lambda, 2 \Delta \lambda$ using the identity:

$$
f^{\prime}(x)=\frac{-\frac{3}{2} f(x)+2 f(x+h)-\frac{1}{2} f(x+2 h)}{h}+O\left(h^{2}\right) .
$$

We consider a numerical application with $\lambda_{\max }=1000 \mathrm{bps}, \Delta \lambda=1 / 5 \mathrm{bps}$ and $\Delta t=1 / 12$. Two approaches have been considered for imposing a boundary value at $\lambda_{\max }$ : either consider that $P_{\Lambda}\left(0, \lambda_{\max }, e_{k}\right)=0, \forall k=1, \ldots, N$ (homogeneous Dirichlet boundary condition) or that $\frac{\partial}{\partial \lambda} P_{\Lambda}\left(0, \lambda_{\max }, e_{k}\right)=0, \forall k=1, \ldots, N$ (homogeneous Neumann boundary condition). Both are correct in the limit $\lambda_{\max } \rightarrow \infty$. We tested the precision of the results by comparing with numerical results obtained on a much larger grid (10 times larger) while using same $\Delta \lambda$. The Neumann boundary condition gives much better results for the situations we considered and as such was always chosen (see also Figure 8.5).

We consider a loan with a contractual maturity $T=5$ years, a nominal amount $K=1$, a recovery rate $\delta=40 \%$. The borrower's default intensity $\lambda_{t}$ follows a CIR dynamics with parameters: initial intensity $\overline{\lambda_{0}}=150 \mathrm{bps}$, volatility $\sigma=0.1$, average intensity $\theta=150 \mathrm{bps}$, reversion coefficient $\gamma=0.5$. We assume a constant interest rate $r=1 \%$ and a liquidity cost defined by a Markov chain of three states $\mathcal{L}_{1}=15$ bps (economic expansion), $\mathcal{L}_{2}=30$ bps (economic stability) and $\mathcal{L}_{3}=250$ bps (recession). The values for the liquidity costs were chosen as follows: we selected a panel of major banks (financing activities in euros) and considered the term structure of the liquidity costs of these banks during the 2007-2008 crisis. The liquidity coefficients $\mathcal{L}_{k}$ were chosen such that the term structure generated by $\mathcal{L}_{k}$ match the one observed. For 
$N=3$ the rate $3 \times 3$ matrix $A$ is defined as following,

$$
A=\left(\begin{array}{ccc}
-\frac{1}{2} & \frac{1}{2} & 0 \\
1 & -2 & 1 \\
0 & \frac{1}{10} & -\frac{1}{10}
\end{array}\right) .
$$

This choice is only an example to support the theoretical results, other choices of matrices $A$ are possible and in practice this should be calibrated from macro-economic data (e.g., for instance GDP or unemployment time-series data). The interested reader can consult Sichel (1994), Hamilton (2005) and related literature; the values given here are compatible (as order of magnitude) with the cited works.

The choice was selected in order to have a matrix that displays inversion of the liquidity term structure. On the other hand the matrix has qualitatively some properties that are often seen in practice:

- the rate of transition from recession to expansion (or vice-versa) is null,

- the rate of transition from stability to either of recession or expansion are of the same order of magnitude,

- the rate of transition from expansion to stability (1/10) is lower than from recession to stability $(1 / 2)$.

We computed the term-structure of the liquidity cost for each state which is plotted in Figure 8.1. At inception, we assume the liquidity cost is in the state $e_{2}$, so $\overline{X_{0}}=e_{2}$. Recall that a basis point, denoted "1 bp" equals $10^{-4}$.

In order to find the initial contractual margin we use equation (4.18) and find $\overline{\rho_{0}}=228 \mathrm{bps}$ at inception in the state $e_{2}$. For information, the contractual margin is $\overline{\rho_{0}}=175 \mathrm{bps}$ in the lowest state $e_{1}$ and $\overline{\rho_{0}}=313 \mathrm{bps}$ in the highest state $e_{3}$. We recall that the contractual margin takes into account the credit risk (default intensity) and the liquidity cost.

The function $\Lambda_{k}^{*}(t)$ is obtained by maximizing $P_{\Lambda}\left(t, \lambda, \overline{X_{0}}\right)$ backward for all $t \in$ $[0, T$ and each state $k$. This is realized numerically in the following way: if the current time step is $(n+1) \Delta t$ then the function $\Lambda_{k}(n \Delta t) \mapsto P_{\Lambda}\left(n \Delta t, \lambda, \overline{X_{0}}\right)$ is maximized with respect to the candidate value $\Lambda_{k}(n \Delta t)$. The maximum will correspond to the optimal boundary $\Lambda_{k}^{*}(n \Delta t)$. This is motivated by the stopping time interpretation that appears in the beginning of the proof of the Theorem 7 . To accelerate the optimization process, for the initial guess at step at $t=T-\Delta t$ we note that there is little time to switch from the current regime to an other. Therefore, we use the optimal boundary for each regime independently (one-regime model), see Figure 8.2, as initial guess. Let $\Upsilon_{k}^{*}(T-\Delta t)$ be the optimal boundary for the constant one-regime $X=e_{k}$ option. We propose as initial guess for $\Lambda^{*}(T-\Delta t)$ the vector $\left(\Upsilon_{k}^{*}(T-\Delta t)\right)_{k=1}^{N}$. This initial guess is validated by computing the value $P_{\Lambda}\left(t, \overline{\lambda_{0}}, \overline{X_{0}}\right)$ for all neighbors around $\left(\Upsilon_{k}^{*}(T-\Delta t)\right)_{k=1}^{N}$ in the $\mathrm{N}$-dimensional space where $\Lambda$ belongs.

Then for each time $t<T-\Delta t$, we search the optimal boundary in the neighborhood of the previous optimal boundary obtained at $t+\Delta t$.

To be accepted, this numerical solution has to verify all conditions of the Theorem 7. The hypothesis (7.8) and (7.10) are satisfied (see Figure 8.5) and the hypothesis (7.10) is accepted after calculation. Moreover $\forall t \in\left[0, T\left[, \Lambda_{1}^{*}(t) \leq\left(\overline{\rho_{0}}-\mathcal{L}_{1}\right) \wedge \bar{\Lambda}_{1}^{0}(t)\right.\right.$ and the analogous holds for $\Lambda_{2}^{*}(t)$.

In the state $\overline{X_{0}}=e_{2}$ and at inception, the present value of cash flows is at par, so $\xi\left(0, \overline{\lambda_{0}}, \overline{X_{0}}\right)=1$. The prepayment option price is $P\left(0, \overline{\lambda_{0}}, \overline{X_{0}}\right)=0.0136$. Therefore the loan value equals $\xi\left(0, \overline{\lambda_{0}}, \overline{X_{0}}\right)-P\left(0, \overline{\lambda_{0}}, \overline{X_{0}}\right)=0.9864$. 


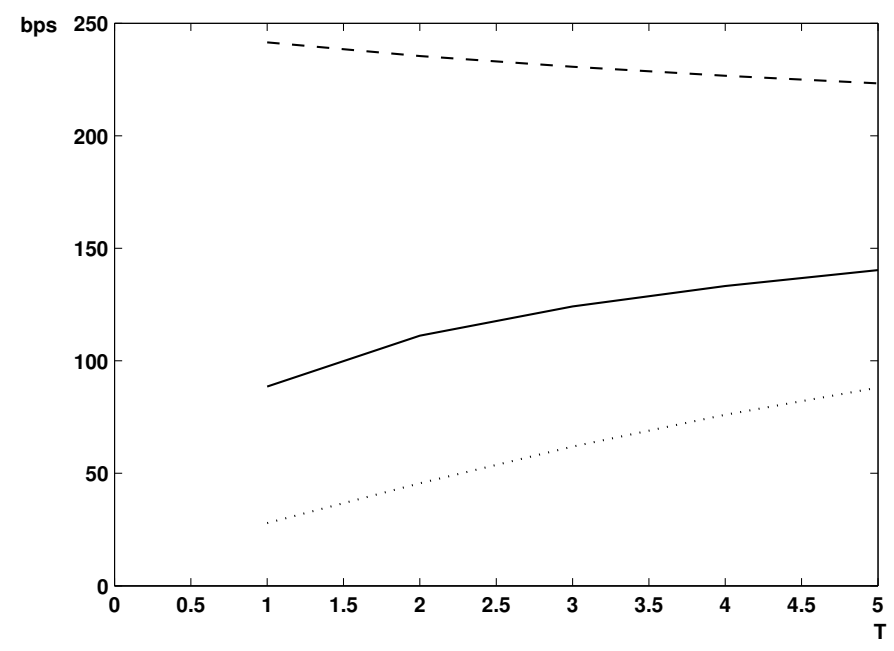

FIG. 8.1. We plot the term-structure of the liquidity cost in bps in the regime $X=e_{1}$ (dashed, recession), $X=e_{2}$ (solid, economic stability) and the regime $X=e_{3}$ (dotted, economic expansion). The curve for the recession regime appears inversed, which trancribes scarcity of short-term money market, in coherence with liquidity prices.

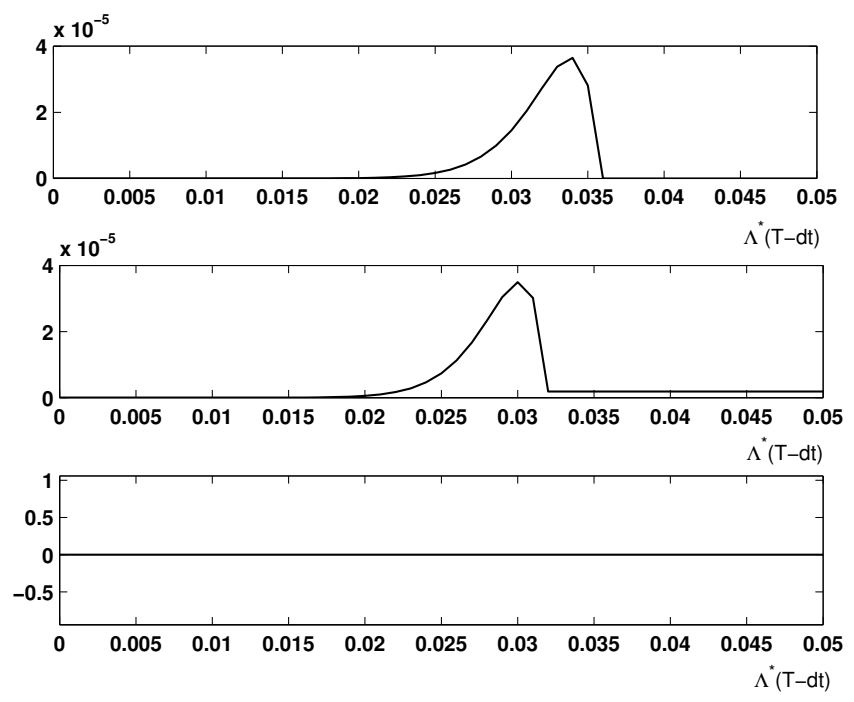

FIG. 8.2. We search for the exercise boundary $\Lambda_{k}^{*}(T-d t)$ that maximizes the option price in the state $X=e_{1}$ (top), $X=e_{2}$ (middle) and $X=e_{3}$ (bottom). We obtain $\Lambda_{1}^{*}(T-d t)=339$ bps, $\Lambda_{2}^{*}(T-d t)=301$ bps and $\Lambda_{2}^{*}(T-d t)=0$ bps. Remark: The state $X=e_{3}$ is a particular case where there exist no exercise boundary since the pay-off is null for all $\lambda>0$.

The loan value will be equal to the nominal if the intensity decreases until the exercise region $\lambda \leq \Lambda^{*}$, see Figure 8.4. The continuation and exercise regions are depicted in Figure 8.5.

The following overall algorithm was implemented:

1. Find the payoff: 


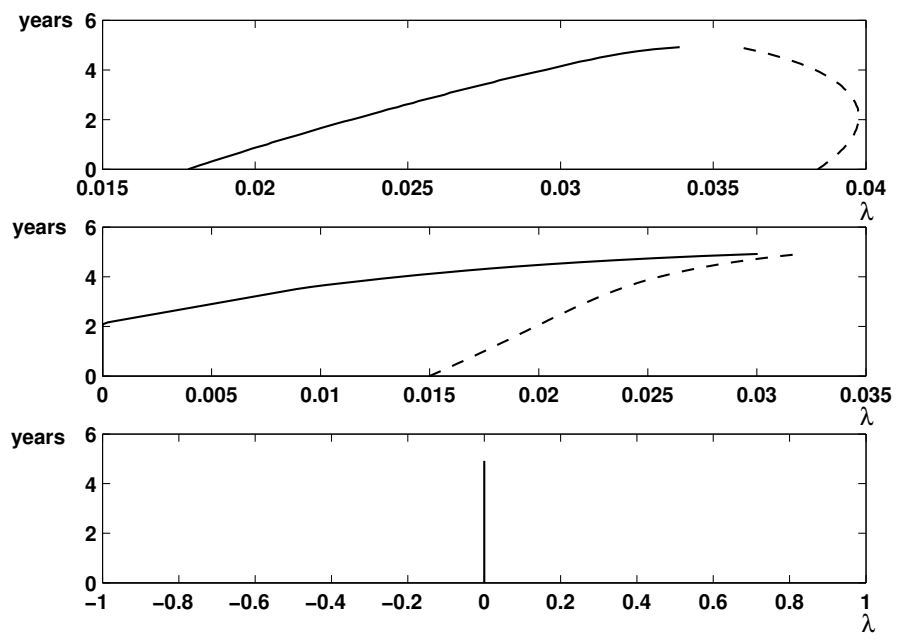

FIG. 8.3. We plot the evolution of the exercise boundary $\Lambda_{k}^{*}(t)$ (solid) that maximizes the option price and the par boundary $\bar{\Lambda}_{k}^{0}(t)$ (dashed) where $\xi(t, \lambda, X)$ verifies the equation (4.19), for all $t \in\left[0, T\left[\right.\right.$ in the state $X=e_{1}$ (top), $X=e_{2}$ (middle) and $X=e_{3}$ (bottom). We obtain $\Lambda_{1}^{*}(0)=178$ bps, $\Lambda_{2}^{*}(0)=0$ and $\Lambda_{3}^{*}(0)=0$ bps. The $x$ axis is $\lambda$ and the $y$-axis is the time.
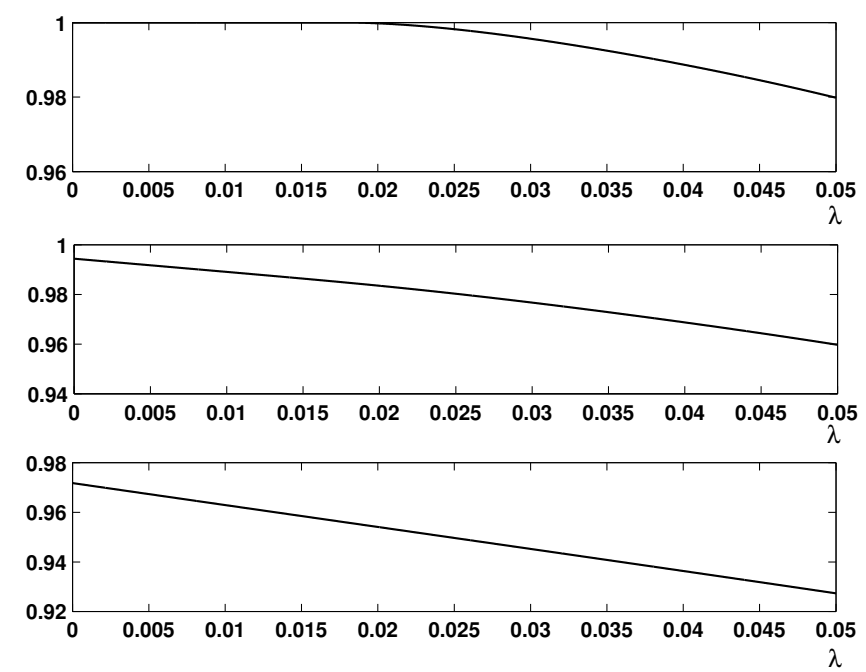

FIG. 8.4. We plot the loan value (at $t=0$ ) as a function of the intensity $\lambda$ in the state $X=e_{1}$ (top), $X=e_{2}$ (middle) and $X=e_{3}$ (bottom). The loan value is decreasing when there is a degradation of the credit quality (that is $\lambda$ increases) and converges to 0.

(a) select the option parameters $A, l, K, T, \sigma, \ldots$;

(b) choose the discretization parameters $\lambda_{\max }, \Delta t, \Delta \lambda$;

(c) start an iterative procedure to compute $\overline{\rho_{0}}$ as the zero of the function of $\overline{\rho_{0}} \mapsto \xi-K$; the procedure is a one-dimensional search that uses the function $\overline{\rho_{0}} \mapsto \xi$. For any given $\overline{\rho_{0}}, \xi$ is computed by solving equation 

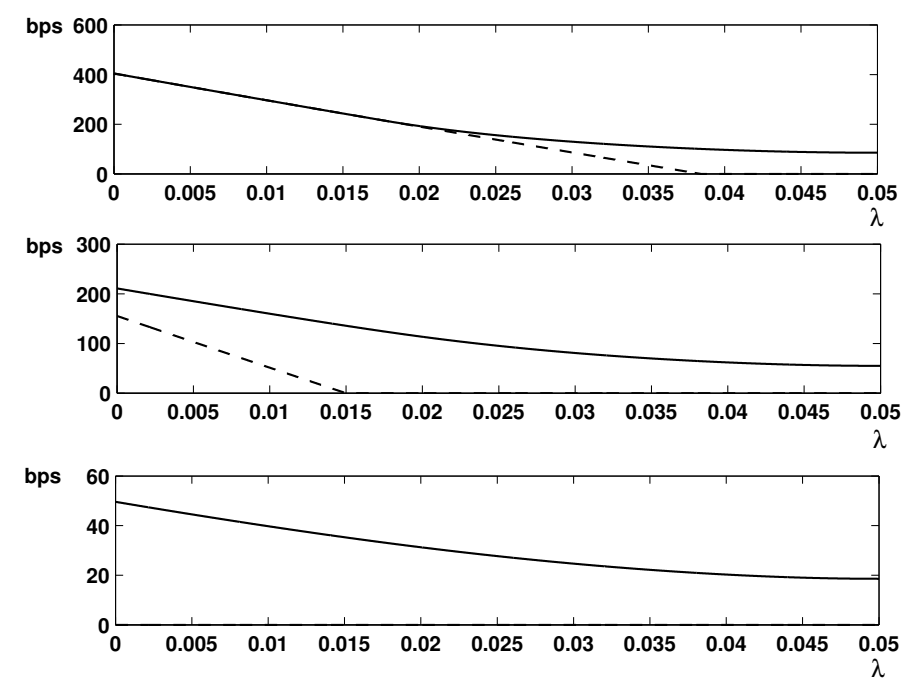

FIG. 8.5. We plot the prepayment option price in bps at inception $P(0, \lambda, X)$ (solid) and pay-off $\chi(0, \lambda, X)$ (dashed) as a function of the intensity $\lambda$ in the state $X=e_{1}$ (top), $X=e_{2}$ (middle) and $X=e_{3}$ (bottom). Two regions appear: the continuation $\lambda>\Lambda_{k}^{*}(t)$ and the exercise region region $\lambda<=\Lambda_{k}^{*}(t)$ except in the third state where there is no exercise region.

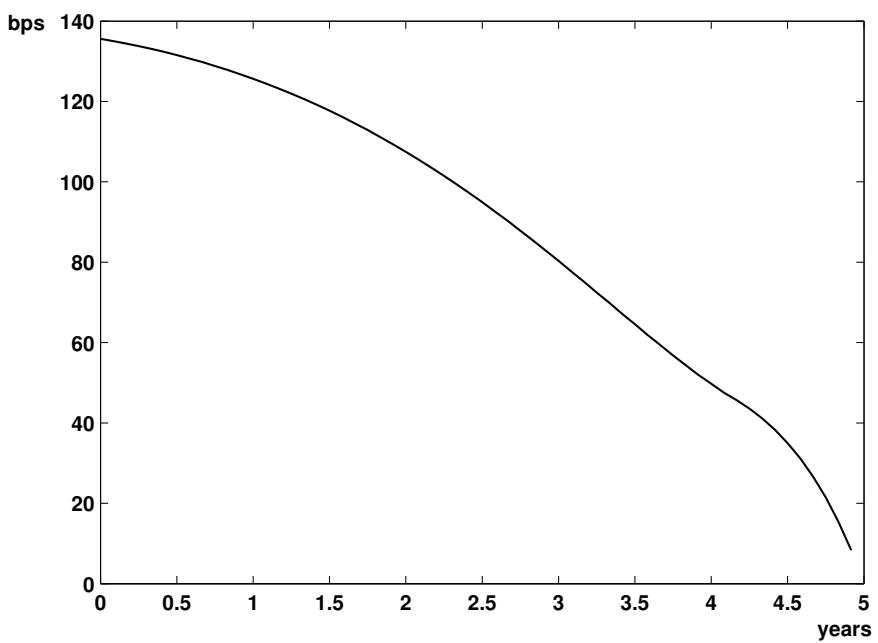

FIG. 8.6. The prepayment option price as a function of the time (the $x$-axis) in the state $X=e_{2}$. As expected the option price converges to 0 when the residual maturity of the loan tends to 0.

(5.2) using the discretization (8.1)-(8.2).

(d) compute the payoff $\chi$ using the $\xi$ corresponding to $\overline{\rho_{0}}$ found above;

2. Numerical Solution to Prepayment Option:

(a) find the optimal exercise boundary $\Lambda^{*}$ as explained above;

(b) check that $\Lambda^{*}$ satisfies the hypothesis of the Theorem 7. In particular the condition (7.9) is checked numerically up to a tolerance depending 
on $\Delta x)$.

3. Find loan value $L V$.

4. For informative reasons, plot the term structure using equations (4.15) and (6.2).

9. Conclusion. The present work proposes a procedure to compute the price of the prepayment option of a corporate loan. The model takes into account a multiregime framework for the liquidity price. The numerical illustration shows that the impact of the option may be non-negligible and should be assessed. Moreover it is seen that the borrower may never prepay during recessions but may do so once the economic situation stabilizes. Although the model does not take into account the correlation between borrowers, it may indicate that the end of a recession will trigger simultaneous prepayments of many loans, putting banks at risk. Appropriate provisions have therefore to be set aside to cope with such an alternative and the present model gives quantitative indications on how to evaluate them.

\section{References.}

Y. Achdou and O. Pironneau. Computational Methods for Option Pricing. Frontiers in Applied Mathematics. Society for Industrial and Applied Mathematics, 2005. ISBN 9780898715736.

A. Alfonsi. On the discretization schemes for the CIR (and Bessel squared) processes. Monte Carlo Methods and Applications, 11(4):355-384, 12 2005. doi: 10.1515/ 156939605777438569.

M. Bardi and I. Capuzzo-Dolcetta. Optimal control and viscosity solutions of Hamilton-Jacobi-Bellman equations. Systems \& Control: Foundations \& Applications. Birkhäuser Boston Inc., Boston, MA, 1997. ISBN 0-8176-3640-4. doi: 10.1007/978-0-8176-4755-1. With appendices by Maurizio Falcone and Pierpaolo Soravia.

G. Barles and J. Burdeau. The Dirichlet problem for semilinear second-order degenerate elliptic equations and applications to stochastic exit time control problems. Communications in Partial Differential Equations, 20(1-2):129-178, 1995. doi: 10.1080/03605309508821090. URL http://www.tandfonline.com/doi/abs/ 10.1080/03605309508821090.

A. Bensoussan and J.-L. Lions. Applications of variational inequalities in stochastic control. Transl. from the French. Studies in Mathematics and its Applications, Vol. 12. Amsterdam - New York - Oxford: North-Holland Publishing Company. XI, 564 p. \$ 82.50; Dfl. 175.00 (1982)., 1982.

J. Buffington and R. Elliott. American options with regime switching. International Journal of Theoretical and Applied Finance, 5:497-514, 2009. ISSN 0219-0249. doi: 10.1142/S0219024902001523.

$\mathrm{X}$. Chen and J. Chadam. A mathematical analysis of the optimal exercise boundary for american put options. SIAM Journal on Mathematical Analysis, 38(5):1613-1641, 2007. doi: 10.1137/S0036141003437708. URL http://epubs.siam.org/doi/abs/ 10.1137/S0036141003437708.

Y. Chen, M. Connolly, W. Tang, and T. Su. The value of mortgage prepayment and default options. Journal of Futures Markets, 29(9):840-861, 2009. ISSN 1096-9934. doi: 10.1002/fut.20388. URL http://dx.doi.org/10.1002/fut. 20388.

D. e. a. Cossin. Pricing prepayment option in C \& I loans at origination. University of Lausanne - School of Economics and Business Administration (HEC-Lausanne), 2002.

J. C. Cox, J. E. Ingersoll, and S. A. Ross. A theory of the term structure of interest rates. Econometrica, 53:385, 1985. 
M. G. Crandall, H. Ishii, and P.-L. Lions. User's guide to viscosity solutions of second order partial differential equations. Bull. Am. Math. Soc., New Ser., 27(1):1-67, 1992. doi: 10.1090/S0273-0979-1992-00266-5.

G. Dionne, G. Gauthier, K. Hammami, M. Maurice, and J.-G. Simonato. A reduced form model of default spreads with Markov-switching macroeconomic factors. Journal of Banking and Finance, 35(8):1984 - 2000, 2011. ISSN 0378-4266. doi: 10.1016/j.jbankfin.2011.01.001. URL http://www.sciencedirect.com/science/ article/pii/S0378426611000227.

J.-C. Duan, J. Sun, and T. Wang. Multiperiod corporate default prediction - A forward intensity approach. Journal of Econometrics, 170(1):191 - 209, 2012. ISSN 0304-4076. doi: http://dx.doi.org/10.1016/j.jeconom.2012.05.002. URL http:// www.sciencedirect.com/science/article/pii/S0304407612001145.

D. Duffie, L. Saita, and K. Wang. Multi-period corporate default prediction with stochastic covariates. Journal of Financial Economics, 83(3):635 - 665, 2007. ISSN 0304-405X. doi: http://dx.doi.org/10.1016/j.jfineco.2005.10.011. URL http:// www.sciencedirect.com/science/article/pii/S0304405X06002029.

R. J. Elliott and T. K. Siu. On Markov-modulated exponential-affine bond price formulae. Applied Mathematical Finance, 16(1):1-15, 2009. doi: 10.1080/ 13504860802015744. URL http://www.tandfonline.com/doi/abs/10.1080/ 13504860802015744.

X. Guo. An explicit solution to an optimal stopping problem with regime switching. J. Appl. Probab., 38(2):464-481, 2001.

J. D. Hamilton. What's real about the business cycle? Working Paper 11161, National Bureau of Economic Research, February 2005. URL http://www.nber.org/ papers/w11161.

J. Hillard, J.E. Kau and V. Slawson. Valuing prepayment and default in a fixed-rate mortgage: A bivariate binomial options pricing technique. Real Estate Economic, 26:431-468, 1998.

Y. Huang, P. A. Forsyth, and G. Labahn. Methods for pricing American options under regime switching. SIAM J. Sci. Comput., 33(5):2144-2168, 2011. ISSN 10648275. doi: DOI:10.1137/110820920. URL http://dx.doi.org/10.1137/110820920.

P. Jaillet, D. Lamberton, and B. Lapeyre. Variational inequalities and the pricing of American options. Acta Applicandae Mathematicae, 21:263-289, 1990. ISSN 01678019. URL http://dx.doi.org/10.1007/BF00047211. 10.1007/BF00047211.

A. Jobert and L. C. G. Rogers. Option pricing with Markov-modulated dynamics. SIAM J. Control Optim, 44(6):2063-2078, 2006. ISSN 03630129. doi: DOI:10.1137/ 050623279. URL http://dx.doi.org/10.1137/050623279.

B. Øksendal. Stochastic differential equations. Universitext. Springer, Berlin ; Heidelberg [u.a.], 6. ed. edition, 2007. ISBN 3-540-04758-1.

D. Lamberton and B. Lapeyre. Introduction to stochastic calculus applied to finance, 2nd edition. Chapman \& Hall/ CRC, 2008.

D. Lando. Credit Risk Modeling. Princeton University Press, 2004.

$\mathrm{X}$. Liang and G. Wang. On a reduced form credit risk model with common shock and regime switching. Insurance: Mathematics and Economics, 51(3):567 - 575, 2012. ISSN 0167-6687. doi: 10.1016/j.insmatheco.2012.07.010. URL http://www . sciencedirect.com/science/article/pii/S0167668712000893.

R. S. Mamon and M. R. Rodrigo. Explicit solutions to European options in a regimeswitching economy. Operations Research Letters, 33:581-586, 2005.

M. Musiela and M. Rutkowski. Martingale Methods in Financial Modelling. Springer, 
2005.

R. Myneni. The pricing of the American option. The Annals of Applied Probability, 2(1):1-23, 1992.

T. Papin and G. Turinici. Valuation of the Prepayment Option of a Perpetual Corporate Loan. Abstract and Applied Analysis, 2013:960789, 2013. doi: 10.1155/2013/960789.

T. Papin and G. Turinici. Prepayment option of a perpetual corporate loan: the impact of the funding costs. International Journal of Theoretical and Applied Finance, 17(04):1450028, June 2014. doi: 10.1142/S0219024914500289.

W. H. Press, S. A. Teukolsky, W. T. Vetterling, and B. P. Flannery. Numerical recipes. The art of scientific computing. 3rd ed. Cambridge: Cambridge University Press, 3rd ed. edition, 2007. ISBN 978-0-521-88068-8/hbk; 978-0-511-33239-5/ebook.

E. Schwartz and W. Torous. Mortgage prepayment and default decisions: A Poisson regression approach. American Real Estate and Urban Economics Association, 21: 431-449, 1993.

D. E. Sichel. Inventories and the three phases of the business cycle. Journal of Business \&5 Economic Statistics, 12(3):269-277, 1994. doi: 10.1080/07350015.1994. 10524542. URL http://amstat.tandfonline.com/doi/abs/10.1080/07350015. 1994.10524542.

T. K. Siu. Bond pricing under a markovian regime-switching jump-augmented Vasicek model via stochastic flows. Applied Mathematics and Computation, 216(11):3184 3190, 2010. ISSN 0096-3003. doi: 10.1016/j.amc.2010.04.037. URL http://www . sciencedirect.com/science/article/pii/S0096300310004637.

T. K. Siu, H. Yang, and J. W. Lau. Pricing currency options under two-factor Markovmodulated stochastic volatility models. Insurance: Mathematics and Economics, 43 (3):295 - 302, 2008. ISSN 0167-6687. doi: 10.1016/j.insmatheco.2008.05.002. URL http://www.sciencedirect.com/science/article/pii/S0167668708000565.

D. D. Yao, Q. Zhang, and X. Y. Zhou. A regime-switching model for European options. In H. Yan, G. Yin, and Q. Zhang, editors, Stochastic Processes, Optimization, and Control Theory: Applications in Financial Engineering, Queueing Networks, and Manufacturing Systems, volume 94 of International Series in Operations Research 63 Management Science, pages 281-300. Springer US, 2006. ISBN 978-0-387-338156.

Q. Zhang and X. Guo. Closed-form solutions for perpetual American put options with regime switching. SIAM J. Appl. Math., 64(6):2034-2049, 2004. ISSN 00361399. doi: DOI:10.1137/S0036139903426083. URL http://dx.doi.org/10. $1137 / \mathrm{S} 0036139903426083$.

N. Zhou and R. Mamon. An accessible implementation of interest rate models with Markov-switching. Expert Systems with Applications, 39(5):4679-4689, 2011. ISSN 0957-4174. doi: 10.1016/j.eswa.2011.09.053. URL http://www.sciencedirect. com/science/article/pii/S0957417411013625. 